\title{
Proteomics: Emerging analytical techniques
}

\author{
Maurya Bharati D. ${ }^{1}$, Pawar Shweta V. ${ }^{1}$, Chate Priya B. ${ }^{2}$, Kayarkar Namita A. ${ }^{1}$, Durgude Suvarna G. \\ ${\text { Boraste } \mathrm{Amol}^{3} \text {, Kadam Pallavi }}^{3}$ and Gomase V.S. ${ }^{4 *}$ \\ ${ }^{1}$ V.E.S. College of Arts, Science and Commerce, Chembur, Mumbai-400 071, India \\ ${ }^{2}$ Bhavan's College, Andheri (west), Mumbai-400 058, India \\ ${ }^{3}$ Padmashree Dr. D.Y. Patil University, Navi Mumbai, 400614, India \\ ${ }^{4 \star}$ School of Technology, S.R.T.M. University, Sub-Centre, Latur, 413512, India
}

\begin{abstract}
Genomic and proteomic approaches together promise to reveal a multidimensional view of a biological system. As compared to Genomic approaches, Proteomic studies have their fair amount of limitations. The technical issues troubling proteomic approaches include gel-to-gel reproducibility, biases toward identifying similar proteins in unrelated proteomic studies, and reliability of protein extraction methods. Many types of information cannot be obtained from the study of genes alone because proteins not genes are responsible for the phenotypes of cells. Mechanisms of disease, aging and the effects of the environment cannot be explained solely by the genome because through the study of proteins can protein modifications be analyzed and the targets of drugs of identified.
\end{abstract}

Keywords-Proteomics, imaging MS, 2D-DIGE, MudPIT, SELDI, MALDI TOF, Capillary electrophoresis

\section{Introduction to Proteomics}

Proteomics is the large-scale analysis of proteins whose major aim is to describe the features of gene products such as protein-protein interactions (PPIs), posttranslational modifications (PTMs), sub cellular localization, protein isoforms, and tissue expression [1,2]. Proteomics, the study of the proteome is a rapidly developing field in which there are numerous new and often expensive technologies, making it of vital importance to use the most appropriate technology for the biological system.[3] Proteome analysis has emerged as a powerful technology to decipher biological processes [4]. The emergence of proteomics, the large-scale analysis of proteins, has been inspired by the realization that the final product of a gene is inherently more complex and closer to function than the gene itself $[5,6]$. Proteins are the functional output of the cell and therefore might be expected to provide the most relevant information. The expression or function of proteins is modulated at many points from transcription to post-translation, which generally cannot be predicted from analysis of nucleic acids alone $[7,9]$. Proteomics has been widespreadly implanted into every field of life science and medicine as an important part of post-genomics era research. The development of theory and technology in proteomics has provided new ideas and research fields for cancer research. Proteomics can be used for not only elucidating the mechanism of carcinogenesis from the whole proteins of the tissue or cell but also seeking the biomarkers for diagnosis and therapy of cancer [10]. This review summarizes and describes the essential characteristics of various proteomic technologies and their roles in cancer research. Proteomics employs mass spectrometry, electrophoresis and chromatography for the detection, identification and characterization of proteins. Mass spectrometry-based proteomics has been the centre of several noteworthy advances in cell biology, particularly in the assignment of the protein composition of cellular organelles [80, 81] and study of protein interaction networks [82]. Mass spectrometry has been widely used to analyze biological samples and became an indispensable tool for proteomics research. Although bottom-up proteomics (analysis of proteolytic peptide mixtures) remains the workhorse for proteomic analysis, middle- and top-down strategies (analysis of longer peptides and intact proteins, respectively) allows more complete characterization of protein isoforms and post-translational modifications. Thus, by combining both the strategies, a proteome can be studied [11]. Among proteomic technologies, matrix-assisted laser desorption ionization timeof-flight mass spectrometry (MALDI-TOF MS) is a technique that has allowed rapid progress in cancer biology. It is a simple and high-throughput technique that analyzes with high sensitivity and specificity intact proteins expressed in complex biological mixtures [12]. MALDI Imaging mass spectrometry (MALDI MSI) is currently popular both for its rapid technological improvements, and for its great potential in high impact bioscience fields [13]. Biological molecules such as proteins, peptides, lipids, xenobiotics, and metabolites can be analyzed in a high-throughput manner. Tissues are analyzed intact and spatial localization of molecules within a tissue is preserved [14]. The gel-based approaches have facilitated vital advances in the measurement of protein expression alterations in normal and disease phenotypic states. Two-dimensional gel electrophoresis (2-DE) with immobilized $\mathrm{pH}$ gradients (IPGs) combined with protein identification by mass spectrometry (MS) is currently the workhorse for proteomics [15]. Consequently, considerable efforts have been devoted to the development of non-gel-based proteome separation technologies to alleviate the 
shortcomings and limitations in 2-D PAGE while reserving the ability to resolve complex protein and peptide mixtures prior to MS analysis. [16] Alternative approaches avoid the use of gels altogether by combining liquid chromatography and mass spectrometry (LC/MS) termed as shotgun proteomics [17]. One chip-based method that has already had a major impact on the discovery of potential biomarkers, especially in cancer research, is surface-enhanced laser desorption/ionisation (SELDI) coupled with timeof-flight mass spectrometry [18]. Capillary electrophoresis (CE) is an electric-field-mediated bioanalytical technique capable of rapid, highresolution separation of very complex sample mixtures [19].

\section{Mass Spectrometry (MS)}

Mass spectrometry is an analytical technique and powerful tool in global proteomic studies [20]. A mass spectrometer analyzes proteins after their conversion to gaseous ions (charged particles), based on their mass-to-charge ratio $(\mathrm{m} / \mathrm{z})$. The important components are: an ion source charging proteins and converting them to gaseous ions, a mass analyzer separating them as a function of their $\mathrm{m} / \mathrm{z}$ ratios, and a detector capturing the ionized proteins after separation. Two methods of ionization are routinely used: matrix-assisted laser desorption ionization (MALDI) uses a laser to desorb and ionize proteins from the solid phase to the gaseous phase, and electrospray ionization (ESI) ionizes and vaporizes proteins from liquid solutions [21]. Different mass spectrometers analyze the mass of peptides in different ways. In time of flight (TOF) instruments peptides fly down a flight tube and the time it takes for the peptide to reach a detector is proportional to the peptide mass. Other mass spectrometers utilise quadropoles, ion traps and Fourier transform ion cyclotron resonance (FTICR) to detect the peptides [22]. Mass spectrometers identifies not only protein sequences, but can also detect post-translational modifications such as acetylations and phosphorylation. Finally, MS can identify important changes in protein profiles associated with clinical features, such as neoplasia, histology, response to chemotherapy, and prognosis [23]. MS can also be used to determine relative levels of expression without the need for prior gel separation. Isotope-coded affinity tags (ICATTM) is a high-throughput MSbased technology that facilitates direct qualitative and quantitative comparisons of complex protein mixtures. Samples to be compared (e.g., cancer versus normal cells) are each labelled with a heavy or light isotope, which couple to cysteine residues of the proteins, the samples are then mixed, proteins enzymatically digested, and peptides analysed by MS. Both the relative abundance of peptides from each sample, and protein identifications can be simultaneously obtained. Other labeling techniques are also available, such as O-water labeling, global internal standard technology (GIST), and isotope tags for relative and absolute quantification (iTRAQTM), each of which has various pros and cons depending on the specific application. Stable isotope labeling with amino acids in cell culture (SILAC) is another quantification strategy for analysis of differential expression between two distinct cellular populations [24]. In order to reduce sample complexity, MS approaches are often coupled to multi-dimensional liquid chromatography (MDLC) prior to the mass spectrometer. It involves the use of a strong cation exchange followed by a reverse phase separation, and the chromatography columns are physically attached on-line to the mass spectrometer. This separates complex protein samples into numerous fractions. Such multidimensional protein identification technology (MudPit) is an attractive approach for analyzing complex samples in a large scale manner, and has been shown to be capable of identifying up to 1484 proteins from yeast in a single experiment [25]. An important advancement in mass spectrometry is the ability of the analyzer to isolate an ion (or peptide) and subject it to further fragmentation into individual amino acids (tandem mass spectrometry, MS/MS). The combination of the same or different mass analyzers allows one to select and fragment ions of interest to determine their structures or in the case of peptides their sequence. This analytical approach is referred to as tandem mass spectrometry or MS/MS. Frequently used mass analyzers are time-of-flights (TOF), quadruple ion traps, linear ion traps, obitraps, and Fouriertransform ion cyclotron resonance (FT-ICR) cells [27]. Recently, a method for determination of phosphorylation sites in proteins has been carried out using MS technology. Phosphorylation is one of the major means of posttranslational regulation of proteins such as p53 and the gap junction protein connexin43 (Cx43). Connexin proteins possess four hydrophobic membrane-spanning domains. The C-terminal region (residues 240-382) of Cx43 appears to be the primary region that becomes phosphorylated. Replacement of Cx43 with a truncated version (Cx43K258Stop) lacking the Cterminal, cytoplasmic tail region yielded mice that died shortly after birth due to an epidermal barrier defect, not the heart defect that is present in Cx43 deficient mice. Thus, considerable evidence indicates that $\mathrm{Cx} 43$ is a highly phosphorylated and a highly regulated protein [26].

Imaging Mass Spectrometry (imaging MS) Imaging MS (IMS) is a powerful technique and an emerging technology that permits the direct 
analysis and determination of the distribution of molecules in tissue sections [87]. It allows spatial localization of many different compounds that are recorded in parallel without the need of a label. Various imaging MS methods, i.e., MALDI and secondary ion MS imaging for direct tissue analysis, can be applied on exactly the same tissue sample which allows the identification of small molecules, peptides and proteins present on the same sample surface. In the proteomics toolbox, it is one of the rapidly developing techniques [28]. It is an emerging technology for discovery of protein signatures which involves the identification of biomarkers by MS directly in tissue biopsies. This technology provides the powerful discovery tool for the investigation of biological processes because the identities of proteins observed do not need to be known in advance. Direct imaging of protein expression in normal and disease tissues has been achieved by in situ MS analysis of tissue sections. Frozen tissue is sliced and sections are applied on a MALDI plate and analysed at regular intervals. The mass spectra obtained at each interval are compared between samples, yielding a spatial distribution of individual masses across the section. Recently, over 100 glioma patients have been analyzed in a reasonably high-throughput manner. Application of direct tissue MALDI-MS to human brain tumours identified protein patterns that distinguished primary gliomas from normal brain tissue and one grade of gliomas from another, with high sensitivity and specificity. In situ MS analysis has also been utilized on samples captured by laser capture micro dissection [29]. Imaging MS is utilized to analyze knockout (KO) mice gene. The Scrapperknockout (SCR-KO) mouse brain showed two types of neurodegenerative pathologies, the spongiform neurodegeneration and shrinkage of neuronal cells. By imaging MS analysis based on principal component analysis (PCA), various changes in the $\mathrm{KO}$ mouse brain were found. Furthermore, information on the position of altered substances can also be known [30]. MALDI IMS was initially applied to tissue sections in 1997 and since then has been applied to a wide variety of different tissues and analytical and clinical problems. Normal invertebrate [31] and mammalian tissues have been examined to determine spatial localization of proteins in different structures. The mouse epididymis has been imaged to identify proteins in specific regions of the organ that may be involved in spermatozoa maturation [32]. Developmental studies have been carried out involving the maturation of mouse prostate [33] and the implantation process of the mouse embryo. A major focus of protein imaging has been in the area of cancer [34, 35] in order to improve molecular classification of grade, helping predict clinical outcome, and examine molecular tumor margins. Moreover, IMS has been used to determine early markers of nephrotoxicity that may result from drug treatment. Recently, IMS has been applied to whole animal sections to examine protein, drug, and metabolite distributions. IMS has also been used to distinguish the various truncated molecular forms of $\beta$-amyloid plaques in a mouse model of Alzheimer's disease. Analysis of human breast carcinoma sections allows visualization of proteins specific to different stages of disease. One of the advantages of IMS is the capability to distinguish molecular species not easily achieved by other techniques. Traditionally, drug distribution analysis has been carried out by the use of autoradiography, which visualizes a radio tag but not the specific molecular species that carries the tag. In contrast, IMS allows for the simultaneous detection of the drug of interest and its metabolites .Confirmation of the identities of these species can be achieved through MS/MS of the compounds of interest directly from the same section. Thus imaging MS has been applied to multiple diseased tissues, including human non-small cell lung tumors, gliomas, and breast tumors. This study indicates that such proteomic information will become important in assessing disease progression, prognosis, and drug efficacy [36].

\section{MALDI TOF}

Matrix-assisted laser desorption/ionization (MALDI) is a soft ionization technique applied in mass spectrometry, allowing the analysis of biomolecules [85]. In this a laser is pulsed at a target surface, containing the sample mixed with a chemical matrix (co-crystallization). The matrix strongly absorbs the laser light energy and subsequently matrix molecules are ejected from the surface, taking some analyte molecules with them into the evacuated chamber. Ionization of the matrix and analyte occurs in the gas phase and ionized particles are then accelerated down the flight tube by an applied voltage and one can choose to accelerate and detect either cations or anions. The mass/charge $(\mathrm{m} / \mathrm{z})$ ratio is calculated by carefully measuring the time it takes for the charged particles to reach the detector after ejection from the source. Particles with larger $\mathrm{m} / \mathrm{z}$ move more slowly down the tube, and thus have a longer time-of-flight. Because the MALDI process essentially favors the production of singly charged molecular ions, it allows the analysis of complex protein mixtures without fractionation. Several characteristics of MALDITOF MS make it a widely used technique for the analysis of complex biological samples such as tissues, whole cells, blood, serum, urine with high mass accuracy far better than any gel system, high-throughput capability (sample analysis in seconds), small required sample size and higher tolerance for salts, buffers, or biological 
contaminants. When used in combination with surface chromatography, this method is also known as surface-enhanced laser desorption/ionization time-of-flight mass spectrometry (SELDI-TOF MS) [37]. In one of the findings , Specific markers for Coxiella burnetii (C.b.) isolates RSA 493, Priscilla, and BUD were detected using matrix-assisted laser desorption/ionization time-of-flight (MALDI-TOF) mass spectrometry (MS) and represents a powerful tool for the rapid, sensitive, and differential characterization of C.b. isolates and is vital for phyloproteomicstudies [38]. MALDI coupled with IMS-TOF MS is used to identify phoshorylated and non-phosphorylated peptides [86]. Recently scientist had demonstrated the application of targeted quantitative proteomics in searching, identifying, and quantifying selected peptides in human cerebrospinal spinal fluid (CSF) using a matrix-assisted laser desorption/ionization time-of-flight tandem mass spectrometer (MALDI TOF/TOF)-based platform. Using the liquid chromatography (LC) MALDI TOF/TOF platform and the complementary identification strategy, they were able to selectively identify and quantify a panel of targeted peptides in the whole proteome of CSF without prior depletion of abundant proteins. Moreover targeted quantitative proteomics to analyze a subset of previously identified candidate markers in CSF samples of patients with Parkinson's disease (PD) at different stages and Alzheimer's disease (AD) along with normal controls had been analyzed [39, 40].

\section{Gel-Based Proteomic Methods}

Quantitative proteomics is the workhorse of the modern proteomics initiative [14]. Gel-based methods are the most commonly used techniques in the proteomic world. 2D gel electrophoresis involves the separation of solubilised proteins in the first dimension according to their charge isoelectric point, followed by their separation in the second dimension by sodium dodecyl sulphatepolyacrylamide gel electrophoresis (SDS-PAGE), according to their relative molecular mass [42]. After locating a spot within the gel, it may be excised, digested and subjected for analysis, typically by matrix-assisted laser desorption/ionization (MALDI) time-of-flight (TOF) MS [43]. 2-DE is currently the only method that can be routinely applied for parallel quantitative expression profiling of large sets of complex protein mixtures such as whole cell lysates, in contrast to promising alternative technologies such as multidimensional protein identification technology, stable isotope labeling that have emerged recently. 2-DE enables the separation of complex mixtures of proteins according to isoelectric point, molecular mass, solubility, and relative abundance. Moreover, it gives a map of intact proteins, which reflects changes in protein expression level, isoforms or post-translational modifications. This is in contrast to liquid chromatography-tandem mass spectrometry based methods, which perform analysis on peptides, where Mr and pl information is lost, and where stable isotope labeling is required for quantitative analysis. Today's 2-DE technology with IPGs has overcome the earlier limitations of carrier ampholyte based 2-DE with respect to reproducibility, and separation of very acidic or basic proteins [44]. The methodological advance in two-dimensional gel electrophoresis (2DGE) has been the multiplexing fluorescent twodimensional fluorescence difference gel electrophoresis (2D-DIGE). 2D-DIGE is based on direct labeling of lysine groups on proteins with cyanine CyDye DIGE Fluor minimal dyes before isoelectric focusing, enabling the labeling of 2-3 samples with different dyes and electrophoresis of all the samples on the same 2D gel. This capability minimizes spot pattern variability and the number of gels in an experiment while providing simple, accurate and reproducible spot matching [45]. Nonetheless, the 2D-DIGE approach offers tremendous promise and has been used increasingly by researchers to address a wide range of biological questions, including substance abuse [50] and other psychiatric illnesses [91-92], oncology [93-94] toxicity [96-97], as well as proteomic profiling in eukaryotic [98-101] and prokaryotic cells [102]. Furthermore 2D-DIGE has been used in postmortem studies of human brain tissue in schizophrenia, bipolar disorder and major depression. A recent study investigated schizophrenia using post-mortem brain tissue at the transcriptome, metabolome and proteome level [49]. Gel- based proteomics helps in analyzing expression of the virulence factors and elucidation of regulatory networks involved in S.aureus virulence which is important for understanding of the virulence potential of pathogen and its interaction with the host [48].

\section{Non-Gel-Based Proteomic Methods}

Gel-based methods have fundamental limitations due to their inability to represent the whole proteome [51]. 2D chromatographic strategy termed multidimensional protein identification technology (MudPIT) has been extensively applied to proteomics analyses, at a peptide level $[67,68]$. It involves digesting the whole protein sample and then separating the peptide mixture by one or two dimensions of chromatography prior to LC-MS analysis [46]. MudPIT strategies when applied to protein mixtures results in a loss of intrinsic information about the intact proteins, this information, which enables the protein identification and a more complete characterization disappears in this case because digestion precedes the separation and the MS 
analyses of the proteins [47]. The separation of intact proteins by liquid chromatography provides many advantages over the MudPIT approach and the use of gel based methods. Liquid chromatography techniques can be used for protein or peptide separations and have recently been improved to handle proteomic analyses of complex samples [66]. Proteins are kept intact throughout the separation and collection, so that several MS-based methods of analysis can be applied, such as the digestion of fractions for protein identification by peptide mass fingerprinting (PMF) or the direct analysis of intact proteins by MS. In both cases, it is possible to more adequately characterize the PTMs of a protein when dealing with an intact protein as the starting point. The use of in-solution protein digestion methods is faster and facile than in-gel digestion and can be easily automated [52].

\section{LC-MS/MS}

Liquid chromatography-mass spectrometry (LC/MS) has emerged a powerful technology in proteomic field in drug discovery, target protein characterization and discovery of biomarkers. Using LC/MS detection of protein posttranslational modifications such as glycosylation and phosphorylation has been analyzed [89]. Sample is separated by one or more dimensions of liquid chromatography to reduce the complexity of peptide fractions and subsequently introduced into a tandem mass spectrometer for sequence-based identification. This technique works better with complex samples than the gelbased methods, generate data faster and have a higher throughput. However, these approaches cannot distinguish between different forms of the same protein due to processes such as posttranslational modification. In addition, stable isotope (ICAT) labeling methods must be combined if differential quantitative analysis of two populations of proteins is needed. This method has been used to study changes in protein expression in a cortical neuron cell culture undergoing DNA damage-induced death, where 150 proteins were identified and quantitated [53].

\section{SELDI}

SELDI is analogus to MALDI. Surface enhanced laser desorption/ionization time-of-flight mass spectrometry (SELDI-TOF MS) is a novel approach that combines two powerful techniques: chromatography and mass spectrometry. [83] In this method, a small volume of a liquid sample, typically a body fluid such as serum, plasma or urine, is spotted onto a chemically modified surface of a special target. SELDI is one of the most used techniques for ability to provide a rapid protein expression profile from a variety of biological and clinical samples and the development of new tools with clinical utility, among them the discovery of new biomarker molecules that could serve as indicators of both physiological and disease states [55]. SELDITOF-MS is comparable to MALDI, the difference being that SELDI uses chromatographic chip arrays to selectively bind subsets of proteins from complex samples. The surfaces can be washed to remove non-specifically bound proteins and substances that can interfere with the ionization process such as salt, detergent. Advantage of this technique involves high throughput via automation and requires minimal sample preparation and the limitation is no direct identification of proteins and sensitive to high molecular weight proteins [56]. The application of SELDI TOF-MS technology is not limited to studies of ovarian, breast, pancreatic and prostate cancers .It has also been used in obtaining proteomic patterns for the diagnosis of bladder cancer from urine samples and cervical cancer from laser capture micro dissected captured cell lysates from tissue samples .Cerebrospinal fluid (CSF) was used in finding and monitoring biomarkers for Alzheimer's disease [54]. In one of the study, surfaceenhanced laser desorption/ionization time-offlight mass spectrometry (SELDI-TOF-MS) was used to analyze proteins associated with T-cell senescence in order to identify potential biomarkers. Clonal populations of T-cells isolated from elderly donors were grown in vitro until senescence, and early passage and late passage (pre-senescent) cells were analyzed using SELDI-TOF-MS technology [84].

\section{Np-RP HPLC}

This technique is used for separation of intact proteins prior to MS analysis. Np-RP HPLC protein separation is highly reproducible and provides intact protein fractions which can be directly analyzed by MALDI-TOF MS for intact molecular weight determination. Np-RP HPLC material takes advantage of fast mass transfer kinetics to provide efficient separation of peptides and proteins, whereas traditional porous packing is often limited by a slow diffusion of biomacromolecules [69]. Non-porous stationary phases were developed in the 1980s [70, 71-73] and have been previously applied for the separation of proteins and peptides by reversed phase chromatography. [74, 75-77, 78, 79]. Overall, np-RP-HPLC followed by MALDI-TOFMS allows for rapid, sensitive, and reproducible protein fractionation and very specific protein characterization by integration of PMF analysis with MS intact molecular weight information [88]. Proteins obtained from a membrane fraction of bovine aortic endothelial cellular lysate were characterized using non porous reversed phase liquid chromatography technique for the fractionation of intact proteins as a separation technique and the analysis of mixture of proteins by MALDI-TOF MS was carried out [57]. 


\section{Capillary Electrophoresis System}

Recently, protein separation and detection techniques have been refined so that they allow proteome screening of biological fluids with good reproducibility and accuracy [58]. In recent years, capillary electrophoresis (CE) has increasingly been used for peptide analysis. Capillary electrophoresis (CE) encompasses a family of related separation techniques that use narrowbore fused-silica capillaries to separate a complex array of large and small molecules. The separation mechanism is based mainly on differences in charge-to-mass ratios, and since peptides are amphoteric, they are ideally suited to electrophoretic analysis [59]. Sample introduction is accomplished by immersing the end of the capillary into a sample vial and applying pressure, vacuum or voltage. Depending on the types of capillary and electrolytes used, the technology of CE can be segmented into several separation techniques. It occurs in a variety of operation modes such as CZE, CEC, CIEF, CITP, CAE, CGE and MEKC that can be combined with MS for tissue and body fluid analysis, particularly urine and cerebrospinal fluid, to identify potential proteomic markers for the clinical diagnosis of many diseases (diabetes mellitus, cancer, arthritis and neurological diseases) and their therapeutic intervention [60]. Capillary Zone Electrophoresis (CZE) is the simplest form of CE. Separation mechanism is based on differences in the charge-to-mass ratio of the analytes. It involves homogeneity of the buffer solution and constant field strength throughout the length of the capillary. Capillary Gel Electrophoresis (CGE) is the adaptation of traditional gel electrophoresis into the capillary using polymers in solution to create a molecular sieve. This allows analytes having similar charge-to-mass ratios to be resolved by size. Capillary Isoelectric Focusing (CIEF) allows amphoteric molecules, such as proteins, to be separated by electrophoresis in a $\mathrm{pH}$ gradient generated between the cathode and anode. A solute will migrate to a point where its net charge is zero. Capillary Electro chromatography (CEC) is a hybrid separation method that couples the high separation efficiency of CZE with HPLC and uses an electric field to run the mobile phase through a packed bed. Because there is minimal backpressure, it is possible to use small-diameter packings and achieve very high efficiencies. Its most useful application appears to be in the form of on-line analyte concentration that can be used to concentrate a given sample prior to separation by CZE. Overall, CE provides a short separation time, low running cost, and a high-resolution technique that requires only a small amount of analyte [90]. Capillary electrophoresis (CE) combined with mass spectrometry (MS) is used for the analysis of intact (i.e., non-digested) proteins. Separations are carried out by CZE and $\mathrm{CIEF}$, whereas electrospray ionization (ESI) and matrix-assisted laser desorption ionization (MALDI) are the widely used ionization methods for interfacing. It is used for analysis of various proteins example protein isoform assignment, single cell analysis, metalloprotein characterization, and biomarker screening. Capillary electrophoresis (CE) provides alternatives to 2-DE for protein separation and alternatives to chromatography for peptide separation [62]. Due to its fast and accurate onestep screening method, CE-MS allows resolution of the urinary small molecular weight proteome in high-throughput mode and also enable assessment of systemic diseases like cardiovascular or infectious diseases [63]. A capillary electrophoresis (CE) using short-end injection approach has been developed for the determination of enzymatic reaction kinetics. Extremely short-effective length of capillary induces reduced analysis times and allows monitoring enzymatic activity by using directly the sample vial as catalysis reactor. Besides, CE presents the advantage of needing very low sample volumes. [61] CE-MS/MS made it possible to discover specific modifications such as ( $\mathrm{N}$-(carboxymethyl)-lysine) in the precise location in the structure of collagen corresponding to posttranslational non-enzymatic modifications. Collagen accumulates reactive metabolites through reactions that are not regulated by enzymes. Collagen was fragmented with cyanogen bromide and then digested with trypsin. This peptide digest was separated by CE, CE-MS/MS, and HPLC-MS/MS. An ion trap MS was used and MS conditions were optimized for both methods [65]. Furthermore, in one of the findings, CE is use to characterize soybean fractions and to analyze soybean proteins and by-products such soybean flour, soybean protein concentrate and soybean protein isolates and their hydrolyzates and a study of their relationships to nutritional, functional and biomedical properties [64].

\section{References}

[1] Pandey A., Mann M. (2000) J Nature; 405:837-846.

[2] Phizicky E., Bastiaens P.I., Zhu H., Snyder M., Fields S. (2000) J Nature; 422:208215.

[3] Olsen J. V., Blagoev B., Gnad F., Macek B., Kumar C., Mortensen P., Mann M. (2006) J Cell.,127:635-648.

[4] Strohman R. (1994) J Biotechnology, 12: 156-164.

[5] Adams M. D., Celniker S. E. Holt R. A. (2000) J Science., 287:2185-2195.

[6] Aebersold R., Rist B. and Gygi S.P. (2000) J Sci., 919:33-47. 
[7] Rosamonde E.Banks, Michael J. Dunn, Prof. Denis F. Hochstrasser, JeanCharles Sanchez (2000) 356 (9243): $1749-1756$.

[8] Valcu C.M., Valcu M. (2007) J Proteome Res., 6: 4677-4683.

[9] Petrak J.,Ivanek R.,Toman O. (2008) J Proteomics., 8: 1744-1749.

[10] Chen Z.C. (1990) J Advances in cancer proteomics., 98:9765-9769.

[11] Gygi S.P., Corthals G.L., Zhang Y., Rochon Y., Aebersold R. (2000) J Sci., 97:93909395.

[12] Aebersold R.H., Leavitt J., Saavedra R.A., Hood L.E. and Kent S.B. (1987) J Sci., 84:6970-6974.

[13] Francese S., Dani F.R., Traldi P., Mastrobuoni G., Pieraccini G., Moneti G. (2009) 12(2):156-174.

[14] Chaurand P., Hayn G., Matter U., Caprioli R.M. (2004) J Zhipu Xuebao.;25:205206, 215.

[15] Gorg A., Weiss W., Dunn M.J. (2004) 4 (12): 3665-85.

[16] Amster J. (1996) J. Mass Spectrom.; 31:1325-1337.

[17] Andersen J. S. and Mann M. (2000) J FEBS Lett:; 480:25-31.

[18] Li J., Zhang Z., Rosenzweig J., Wang Y.Y., Chan D.W. (2002) J Clin Chem.,48 (8): 1296-304.

[19] S. Mittermayr, Olajos M., Chovan T., Bonn G.K. and Guttman A. (2008).

[20] Eckel-passow J.E., Oberg A. L., Therneau T. M., Bergen H.R. (2009) An insight into high-resolution mass-spectrometry data Biostatistics; 10(20) 481-500.

[21] Biemann K. (1988) J Mass Spectrom, 16:99-100.

[22] Amster J. (1996) J Mass Spectrom, 31:1325-1337.

[23] Cash P. (2000) J Electrophoresis, 21:11871201.

[24] O'Farrell P. H. (1975) J. Biol. Chem., 250:4007-4021.

[25] Opiteck G. J., Lewis. K.C., Jorgenson. J. W. and Anderegg R. J. (1997) J Anal. Chem., 69:1518-1524.

[26] Crow D.S., Beyer E.C., Paul D.L., Kobe S.S., Lau A.F (1990) J Mol Cell Biol., 10:1754-1763.

[27] Opiteck G. J., Lewis K.C., Jorgenson J.W. and Anderegg R.J. (1997) J Anal. Chem., 69:1518-1524.

[28] Kaletas B.K., van der Wiel I. M., Stauber J., Lennard J. Dekker., Güzel C., Kros J.M., Luider T. M., Heeren R. M. Sample preparation issues for tissue imaging by imaging $M S$.

[29] Chaurand P., Stoeckli M., Caprioli R.M. (1999) J Anal Chem.,71(23):5263-5270.
[30] Yao I., Sugiura Y., Matsumoto M., Setou M. In situ proteomics with imaging mass spectrometry and principal component analysis in the Scrapper-knockout mouse brain.

[31] Kruse R., Sweedler J.V. (2003) J Am Soc Mass Spectrom, 14:752-759.

[32] Chaurand P. (2003) J Proteomics, 3:22212239.

[33] Chaurand P. (2008) J Mol Cell Proteomics, 7:411-423.

[34] Caldwell R.L., Gonzalez A., Oppenheimer S.R., Schwartz H.S., Caprioli R.M. (2006) J Cancer Genomics Proteomics., 3:279-288.

[35] Amann J. M. (2006) J Clin Cancer Res., 12:5142-5150.

[36] Chaurand P., Sanders M.E., Jensen R.A., Caprioli R.M. (2004) Mass Spectrometry Research Center, 165(4):1057-68.

[37] Jonscher, K.R., and J.R. Yates III. (1997) J Anal. Biochem, 244:1-15.

[38] Skultéty L., Hernychová L., Beregházyová E., Slabá K., Toman R. Detection of specific spectral markers of Coxiella burnetii isolates by MALDI-TOF-MS.

[39] Pan S., Rush J., Peskind E.R., Galasko D., Chung K., Quinn J. (2008) Department of Pathology. 7(2):720-30.

[40] McCormack A.L., Schieltz D.M., Goode B., Yang S., Barnes G., Drubin D., Yates J.R. (1997) J Anal. Chem., 69:767.

[41] O'Farrell P. (1975) J. Biol. Chem., 250:4007.

[42] Check E. (2004) J Nature, 429, 496 -497.

[43] Klose J. Humangenetik. (1975) 26:231.

[44] Scheele G.A. (1975) J. Biol. Chem., 250:5375.

[45] O'Farrell P. H. (1975) J Biol Chem., 250:4007-4021.

[46] Bjellqvist B. (1982) J Biochem Biophys Methods., 6:317-339.

[47] Fujii K., Nakano T., Kawamura T., Usai F. (2004) J Proteome Res., 3:712-718.

[48] Engelmann S., Hecker M. (2009) 6:187197.

[49] Edgar P. F., Schonberger S. J., Dean B. (1999) J Molecular Psychiatry., 4, 173 178.

[50] Lin H., Natan M., Keating C. (2000) J Anal. Chem., 72, 5348-5355.

[51] Johnson M. D., Yu L. R., Conrads T. P. (2004) J Cell., 279, 26685 -26697.

[52] Johnston-Wilson., N.L.Sims, C. D.Hofmann J P. (2000) J Molecular Psychiatry.,5, 142-149.

[53] Jacobs I. J., Menon U. (2004) J Mol Cell Proteomics.,3:355-66.

[54] Bertucci F., Birnbaum D., Goncalves A. (2006) J Mol Cell Proteomics.,5:177286. 
[55] Espejel F., Roa J.C. (2008) J Proteomics, 131(8):312-7.

[56] Bergeron J.J. \& Hallett M. (2007) J Nat. Biotechnology., 25, 61-62.

[57] Retamal C.A., Thiebaut P., Alves E.W. (1999) J Anal Biochem, 268:15-20.

[58] Foley J.P. (1990) J Anal. Chem., 62, 1302.

[59] Haselberg R., de Jong G.J., Somsen G.W. Capillary electrophoresis-mass spectrometry for the analysis of intact proteins.Department of Biomedical Analysis.

[60] Farid E.Ahmed (2008) Department of Radiation Oncology, Cancer Center, USA

[61] Marzell Herold, Gordon A. Ross, Rudolf Grimm, David N. Heiger. Capillary electrophoresis using short end injection method for enzyme kinetics reaction.

[62] Haselberg R., de Jong G.J., Somsen G.W. (2007) Capillary electrophoresis-mass spectrometry for the analysis of intact proteins, 3:1159(1-2).

[63] Metzger J., Schanstra J.P., Mischak H. (2009) Capillary electrophoresis-mass spectrometry; 393(5):1431-42.

[64] Saz J. M., Marina M. L. Electrophoresis in the analysis of soybean proteins and peptides in foodstuffs, Spain.

[65] Rodrigues M.R.A., Caramao E.B., Arce L., Rios A., Valcarcel M. (2002) J. Agric. Food Chem., 50, 4215.

[66] Neverova I., Van Eyk J.E. (2005) J Chromatogram B Analyt Technol Biomed Life Sci., 815:51-63.

[67] Uetz P.L., Giot G., Cagney . (2000) J Nature., 403:623-627.

[68] Coldham N.G., Woodward M. J. (2004) J Proteome Res., 3:595-603.

[69] Banks J.F., Gulcicek E.E. (1997) J Anal Chem., 69:3973-3978.

[70] Maa Y.F., Horvath C. (1988) J Chromatogr., 445:71-86.

[71] Jilge G., Janzen R., Giesche H., Unger K.K. (1987) J Chromatogr., 397:71-80.

[72] Janzen R., Unger K.K., Giesche H., Kinkel J.N., Hearn M.T. (1987) J Chromatogr., 397:81-89.

[73] Wall D. B., Lubman D.M., Flynn S.J. (1999) J Anal Chem., 71:3894-3900.

[74] Banks J.F., Gulcicek E.E. (1997) J Anal Chem., 69:3973-3978.

[75] Chen Y., Wall D., Lubman D.M. (1998) J Rapid Commun Mass Spectrom., 12:1994-2003.

[76] Lee W.C. (1997) J Chromatogr B Biomed Sci Appl., 699:29-45.

[77] Itoh H., Nimura N., Kinoshita T., Nagae N., Nimura M. (1991) J Anal Biochem.,199:7-10.
[78] O'Neil K.A., Miller F.R., Barder T. J., Lubman D.M. (2003) J Proteomics; 3:1256-1269.

[79] Cronshaw J.M., Krutchinsky A.N., Zhang W., Chait B.T., \& Matunis M.J. (2002) J. Cell Biol. 158, 915-927.

[80] Au C.E. (2007) J Cell Biol 19, 376-385.

[81] Yates J.R. III., Gilchrist A., Howell K.E., \& Bergeron J.J. (2005) J Nat. Rev. Mol. Cell Biol. 6, 702-714.

[82] Collins S.R. (2007) J Mol. Cell. Proteomics; 6, 439-450.

[83] Terabe S., Otsuka K., Ichikawa K., Tsuchiya A., Ando T. (1984) J Anal. Chem.,56, 111.

[84] Dawn J. Mazzatti , Graham Pawelec, Robin Longdin, Jonathan R. Powell and Rosalyn J. Forsey. (2007) J Proteome Science.,10.1186/1477-5956-5-7.

[85] Ruotolo (2004) J Anal.Chem., 76:67276733.

[86] Karas M., Bahr U. (1990) J Trends Anal. Chem., 9: 321 1016-9936.

[87] McDonnell L.A., Heeren R.M.A. (2001) J Mass Spectrom, 26:606-643.

[88] Dauly C., Perlman D.H., Costello C.E., McComb M.E.(2006) J Proteome Res., 5(7):1688-1700.

[89] Chen G., Pramanik B.N. (2009) J Drug Discovery., 14(9-10):465-71.

[90] Marzell Herold, Gordon A. Ross, Rudolf Grimm, David N. Heiger (2004) J Mol Cell Proteomics, 25(23-24):3913-26.

[91] Hu Y., Malone J.P., Fagan A.M., Townsend R.R., Holtzman D.M. (2005) J Mol Cell Proteomics., 4:2000-2009.

[92] Karp N.A., Griffin J.L., Lilley K.S. (2005) J Proteomics, 5:81-90.

[93] Swatton J.E., Prabakaran S., Karp N.A., Lilley K.S., Bahn S. (2004) J Mol Psychiatry., 9:128-143.

[94] Seike M. (2005) J Proteomics., 5:29392948.

[95] Somiari R.I., Somiari S., Russell S., Shriver C.D. (2005) J Chromatogram B Anal Technol Biomed Life Sci., 815:215-225.

[96] Seike M. (2004) J Proteomics.,4:2776-2788.

[97] Somiari R.I. (2003) J Proteomics., 3:18631873.

[98] Lee I.N. (2005) J Proteome Res., 4:20622069.

[99] Alm H. (2006) J Environ Health Perspect, 114:254-259.

[100] Roelens S.A. (2005) J Ann NY Acad Sci.; 1040:454-456.

[101] Kleno T. G. (2004) J Proteomics; 4:868880.

[102] Tian Q. (2004) J Mol Cell Proteomics. 3:960-969. 